\title{
The Influence of Agreeableness on Leadership in Normal University's Undergraduates: A Moderated Mediation Model
}

\author{
Fei Guoai", You Qingxiu \\ School of Education science, Shanxi Normal University, Linfen, China \\ Email address: \\ psyChilli@163.com (Fei Guoai), 16635711129@163.com (You Qingxiu) \\ ${ }^{*}$ Corresponding author
}

\section{To cite this article:}

Fei Guoai, You Qingxiu. The Influence of Agreeableness on Leadership in Normal University's Undergraduates: A Moderated Mediation Model. Science Innovation. Vol. 8, No. 3, 2020, pp. 70-75. doi: 10.11648/j.si.20200803.14

Received: April 14, 2020; Accepted: May 26, 2020; Published: June 8, 2020

\begin{abstract}
In order to clarify the mechanism of agreeableness on leadership, this study constructed a moderated mediation model, a questionnaire survey was conducted on 426 postgraduates from a normal university in Shanxi Province to investigate the effects of agreeableness on leadership, the mediating role of self-efficacy, and the moderating role of empathy in the direct and indirect relationship between agreeableness and leadership. The results indicated that: (1) Agreeableness have significant positive predictive effects on self-efficacy and leadership.(2) Self-efficacy plays a partial mediating role between agreeableness and leadership. Agreeableness can not only directly predict postgraduates' leadership, but also indirectly predict leadership through the self-efficacy. (3) Empathy plays moderator role in the direct effect and the first half of mediating effects. Lower empathy in direct pathways can increase the impact of agreeableness on leadership. In the mediation path, self-efficacy has a greater impact on leadership at low levels of empathy. The research results are not only conducive to understanding the relationship between agreeableness and college students' leadership from the perspectives of self-efficacy theory and trait theories leadership, but also of great significance to the cultivation of college students' leadership, professional qualities and teaching quality in China's normal universities.
\end{abstract}

Keywords: Agreeableness, Leadership, Self-efficacy, Empathy

\section{宜人性特质对师范院校大学生领导力的影响: 一个有调节的中介 模型}

\author{
费国爱"，尤清秀 \\ 山西师范大学教育科学学院, 临汾, 中国
}

邮箱

psyChilli@163.com（费国爱），16635711129@163.com（尤清秀）

\begin{abstract}
摘要: 为明确宜人性特质对领导力的作用机制, 本研究构建了一个有调节的中介模型, 以问卷法对山西省某师范院校 的520名在校大学生进行调查, 考察宜人性特质对大学生领导力的影响, 同时检验自我效能感在宜人性特质与领导力之 间的中介作用以及共情对该中介路径的调节作用。结果表明：（1）宜人性特质对自我效能感和领导力都有显著的正向 预测作用; (2) 自我效能感在宜人性特质与领导力之间起着部分中介作用, 宜人性不仅能直接预测大学生领导力, 还 可以通过自我效能感间接预测领导力; (3) 共情对直接效应及中介效应的前半段路径起着调节作用, 在直接路径中低 共情会加大宜人性对领导力的影响, 在中介路径中, 低共情会加大宜人性对自我效能感的影响。研究结果不仅有利于 从自我效能感理论和领导特质理论的视角理解宜人性与大学生领力的关系, 而且对我国师范院校大学生领导力的培养、 职业素养和教学质量的提升都具有重要意义。
\end{abstract}


关键词: 宜人性, 领导力, 自我效能感, 共情

\section{1. 引言}

人才的领导力越来越受到重视, 研究者们普遍认为领 导力是每个人都需要培养的重要技能 $[1,2,3]$,因此领导 力也越来越受到学校教育的关注, 开始把其视为一种能力 素质而进行面向所有学生的覆盖式教育。大学生领导力是 一种在各种正式和非正式领导情境中能够吸引和影响他 人以实现目标并对活动效果产生影响的能力和素质的组 合 [4]。大学阶段是培养领导素质的关键期, 对大学生进行 领导教育顺应了全球化的时代要求和领导人才的成长规 律 [5], 因此深入探究大学生领导力的影响机制, 对提升大 学生领导力具有重大而深远的意义。

师范院校大学生是我国教师的储备力量, 他们作为 未来教育发展中最重要的人力资源力量, 其领导力能够 在日常交往中潜移默化地影响学生, 且直接影响学校人 才培养的质量和效能[6]。研究表明, 发展自我和教导学 生是每一位教师必须具备的基础层级的领导核心要素, 而引领同伴和参谋领导则是优秀教师所具备的领导核心 要素, 教师的领导力体现在其职业活动中 [7]。因此, 在 教学过程中, 教师可以被看作是一个领导者, 教学本身 包含着领导的内在特质, 也印证了服务型教师领导力正 向影响学生的学习投入以及教师领导力直接影响师生关 系的研究 [8,9]。因此, 对师范院校大学生的领导力进行 探讨可以为我国教师的职业适应和发展提供参考, 并有 望进一步提升教学质量。

宜人性是大五人格之一, 宜人性个体具有信任他人, 顺从和关心他人，慷慨助人、谦逊和合作等特点 $[10]$ 。根 据领导特质理论, 领导者的某些个性特质是与生俱来的, 领导者自身一些独特的、能与他人区别开来的品质或特质 会对领导力产生影响[11]。元分析的证据表明大五人格特 质中外倾性、开放性和尽责性三特质与领导力正相关，而 神经质特质与领导力负相关, 只有宜人性特质的作用尚未 表现出明显的相关趋势 [12]; 而王超等人的结果表明东西 文化差异调节了宜人性和企业家成就之间的关系[13]。因 此, 在我国文化更加注重团结与和谐的背景下, 宜人性特 质可能与领导力正相关, 而教师相较于其他职业更需要表 现出对学生的关怀, 更需要以“师爱”为基础, 即宜人性特 质可以正向预测师范院校大学生领导力。

在领导力的众多影响因素中, 自我效能感也起着重要 作用。研究证实, 宜人性特质对效能感有着极其显著的正 向预测作用 [14]。宜人性较强的个体更加富有同情心且有 较强的合作精神, 这意味着该类个体更容易被人接纳而较 少与他人产生冲突, 因此表现出较高的自我效能感, 能够 克服各种苦难和挑战[15], 进而对领导力产生正向的影响。 根据自我效能理论, 自我效能感会为行为主体提供具体的 行为动机, 促进实施特定的行动和绩效目标达成 [16]。由 此可以推测宜人性较高的个体, 其自我效能感越强, 进而 越可能对领导力产生积极影响, 即自我效能感在宜人性特 质和领导力之间起中介作用。
共情能力也是重的影响因素, 其高低能直接体现个体 对他人和社会的接纳程度和被接纳的程度。宜人性人格的 个体能进行更好的归因和更高的共情[17]; 共情能力不同 的个体对自我效能感的影响存在差异, 大学生的共情能力 越高, 其自我效能感越强且共情的所有子维度都与领导力 技能呈显著正相关 [18，19]。据此可以推断，共情作为一 种调节性因子将更容易影响宜人性或自我效能感对领导 力的作用。模型假设如图1所示。

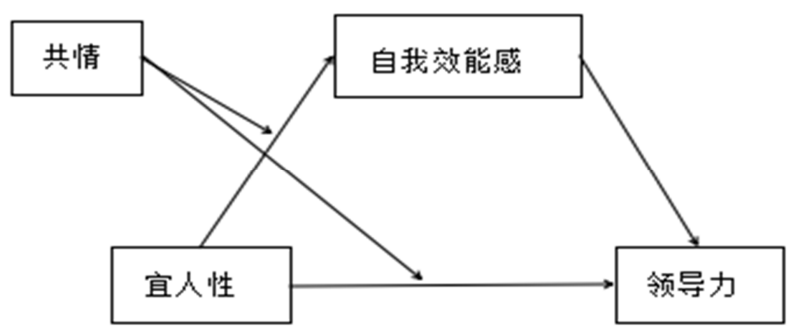

图1 宜人性、自我效能感、共情与领导力关系的假设模型。

\section{2. 方法}

\section{1. 被试}

采取随机取样的方法对山西省某师范大学的568名在 校大学生进行调查分析, 回收有效问卷520份, 问卷回收 率为 $91.5 \%$, 其中男生 108 人 $(20.8 \%)$, 女生 412 人 $(79.2 \%)$, 年龄18 24岁, 平均年龄20.68岁（ $\mathrm{SD}=1.57 ） 。$

\section{2. 工具}

\subsection{1. 宜人性分量表}

采用王孟成等编制的中国大五人格量表简式版 (CBF-PI-B) 的宜人性分量表, 共 8 个题目, 采用 5 点记分, 分数越高代表个体的宜人性特质越强, 问卷 Cronbach $\alpha=0.707$ 。

\subsection{2. 一般自我效能感量表}

采用Schwarzer等编制, 王才康等人进行翻译修订的一 般自我效能感量表 (GSES), 共10个题目, 采用 4 点记分, 得分越高代表自我效能感水平越高。问卷 Cronbach $\alpha=0.890$ 。

\subsection{3. 人际反应指针问卷}

采用Davis（1980）编制，并由詹志禹等人（1987） 进行修订的中文版人际反应指针问卷（IRI-C），共 22 个 题目, 分为观点采择 (PT)、幻想 (FS)、共情关心 (EC) 和个人痛苦 (PD) 四个因子, 采用 5 点记分, 得分越高说 明个体的共情能力越强。问卷Cronbach $\alpha=0.819$ 。 


\subsection{4. 大学生领导力量表}

采用我国学者张超 (2012) 编制的大学生领导力量表, 共有 20 个题目, 分为任务处理、关系处理、动机态度三个 因子, 采用 5 点记分, 得分越高表示个体领导力越强。问 卷Cronbach $\alpha=0.917$ 。

\section{3. 统计方法}

采用SPSS22.0进行数据前期处理、描述性统计、各变 量之间的相关分析和信度分析; 采用Process检验自我效能 感在宜人性特质和领导力之间的中介作用, 以及共情的调 节作用; 所有回归系数的显著性检验均采用Bootstrap方法 进行。

\section{3. 结果}

\section{1. 共同方差偏差检验}

为避免共同方法偏差对研究结果的影响,采用Harman 单因子检验对原始数据进行共同方法偏差检验。结果显示, 特征根大于 1 的因子共有 12 个, 第一公因子的解释率为 $22.68 \%$, 小于 $40 \%$ 的临界值, 因此认为不存在严重的共同 方法偏差。

\section{2. 大学生宜人性、效能感、共情和领导力的均值、标 准差和相关矩阵}

表1 各变量的均值、标准差和相关矩阵 $(n=520)$ 。

\begin{tabular}{llllll}
\hline & $\mathbf{M} \pm \mathbf{S D}$ & $\mathbf{1}$ & $\mathbf{2}$ & $\mathbf{3}$ & $\mathbf{4}$ \\
\hline 1 宜人性 & $28.22 \pm 4.56$ & 1 & & & \\
2 自我效能感 & $24.08 \pm 5.28$ & $0.18^{* *}$ & 1 & & \\
3 共情 & $72.34 \pm 10.15$ & $0.44^{* *}$ & $0.12^{*}$ & 1 & \\
4 领导力 & $68.42 \pm 11.53$ & $0.33^{* *}$ & $0.67^{* *}$ & $0.37^{* *}$ & 1 \\
\hline
\end{tabular}

注: (M为平均数, $\mathrm{SD}$ 为标准差; ${ }^{*} p<0.05, * * p<0.01, * * * p<0.001$, 下同)。

如表1所示, 宜人性特质与领导力呈显著正相关, 与 自我效能感显著正相关, 且自我效能感与领导力呈显著正 相关。共情与自我效能感、宜人性、领导力均呈显著正相 关。研究变量两两之间相关显著, 符合调节效应的检验的 条件[20], 接下来可以继续进行有调节的中介检验。

\section{3. 宜人性特质与师范院校硕士生领导力的关系: 有调 节的中介模型检验}

根据Hayes和Scharkow (2013)、温忠麟和叶宝娟 (2014) 的观点, 检验有调节的中介模型需要对 3 个回归方程进行 估计, 参数计算均通过SPSS中的PROCESS2.16插件完成。 为了避免多重共线性, 先对所有变量进行标准化处理; 同 时将性别、年级作为协变量进行了控制。方程 1 估计调节 变量对自变量和因变量之间关系的调节效应; 方程 2 估计 调节变量对自变量和中介变量之间关系的调节效应; 方程 3 估计中介变量对自变量和因变量之间关系的中介作用。 各方程的参数估计结果见表 2 。

表2 有调节的中介效应检验（方程1、2、3, $n=520 ） 。$

\begin{tabular}{|c|c|c|c|c|c|c|c|c|c|c|c|c|}
\hline \multirow{2}{*}{ 预测变量 } & \multicolumn{4}{|c|}{ 方程1（Y：领导力） } & \multicolumn{4}{|c|}{ 方程2（M: 效能感） } & \multicolumn{4}{|c|}{ 方程3（Y: 领导力） } \\
\hline & $B$ & $t$ & $p$ & $95 \% C I$ & $B$ & $t$ & $p$ & $95 \% C I$ & $B$ & $t$ & $p$ & $95 \% C I$ \\
\hline 宜人性 & $0.19^{* * *}$ & 3.80 & $<0.001$ & {$[0.09,0.28]$} & $0.13^{*}$ & 2.49 & $<0.01$ & {$[0.03,0.24]$} & $0.22^{* * *}$ & 6.2 & $<0.001$ & {$[0.15,0.29]$} \\
\hline 共情 & $0.28^{* * *}$ & 5.69 & $<0.001$ & {$[0,18,0.37]$} & 0.06 & 1.05 & 0.29 & {$[-0.05,0.16]$} & & & & \\
\hline 宜人性*共情 & $-0.13^{* *}$ & -3.20 & 0.002 & {$[-0.21,-0.05]$} & $-0.10^{*}$ & -2.28 & 0.02 & {$[-0.18,-0,01]$} & & & & \\
\hline 效能感 & & & & & & & & & $0.63^{* * *}$ & 17.94 & $<0.001$ & {$[0.56,0.70]$} \\
\hline 常数项 & $69.09^{* * *}$ & 141.99 & $<0.001$ & {$[68.13,70.04]$} & $32.16^{* * *}$ & 102.25 & $<0.001$ & {$[31.55,32.78]$} & $15.95^{* * *}$ & 6.24 & $<0.001$ & {$[10.93,20.98]$} \\
\hline$R^{2}$ & $0.19^{* * *}$ & & & & $0.04^{* * *}$ & & & & $0.49^{* * *}$ & & & \\
\hline$F$ & 33.14 & & & & 6.62 & & & & 206.96 & & & \\
\hline
\end{tabular}

首先, 回归方程 1 显示宜人性特质和共情能显著预测 领导力, 而宜人性特质和共情的交互项对领导力的预测作 用显著, 说明共情在宜人性特质对领导力的影响过程中起 调节作用。回归方程 2 和 3 显示宜人性特质与共情的交互项 对自我效能感的预测作用显著, 宜人性特质对自我效能感 的预测作用显著, 说明共情在宜人性对自我效能感的影响 过程中起调节作用。与此同时, 宜人性特质对领导力预测 作用显著, 且自我效能感对领导力的主效应显著, 说明自 我效能感在宜人性对领导力的影响过程中起中介作用。

综合以上结果，宜人性特质、自我效能感、共情、领 导力四者之间构成了一个有调节的中介效应模型, 自我效 能感在宜人性特质和领导力之间起中介作用, 共情在模型 的前半路径和直接路径上起调节作用。

共情的调节效应模型是一个既调节了直接效应, 又调 节了中介效应前半段的模型。为进一步解释有调节的 中 介模型, 将宜人性按照正负一个标准差分成高、低两组,
通过用简单斜率检验考察在不同共情水平上宜人性特质 对领导力的影响以及宜人性对自我效能感的影响。具体的 调节效应如图2和图3所示。

图2表明, 在共情能力较低时, 随着宜人性特质的增 加, 领导力表现出显著的上升趋势 $\left(B_{\text {simple }}=0.31, t=5.41\right.$, $p<0.001$ ) ; 在共情能力较高时, 随着宜人性特质的增加, 领导力的上升趋势变得不显著 $\left(B_{\text {simple }}=0.06, t=0.88\right.$, $p>0.05)$, 相比低共情时, 增加幅度明显变小。图3表明, 当共情能力较高时, 宜人性特质对自我效能感的影响不显 著 $\left(B_{\text {simple }}=0.07, t=0.75, p>0.05\right)$; 当共情能力较低时, 宜人性特质对自我效能感的影响显著 $\left(B_{\text {simple }}=0.33, t=3.99\right.$, $p<0.001)$ 。相比高共情能力时, 增加幅度明显变大。

分析宜人性对领导力的条件中介效应发现，宜人性对 领导力的直接效应为 $0.22,95 \%$ 置信区间为 $[0.15,0.29]$ 。 


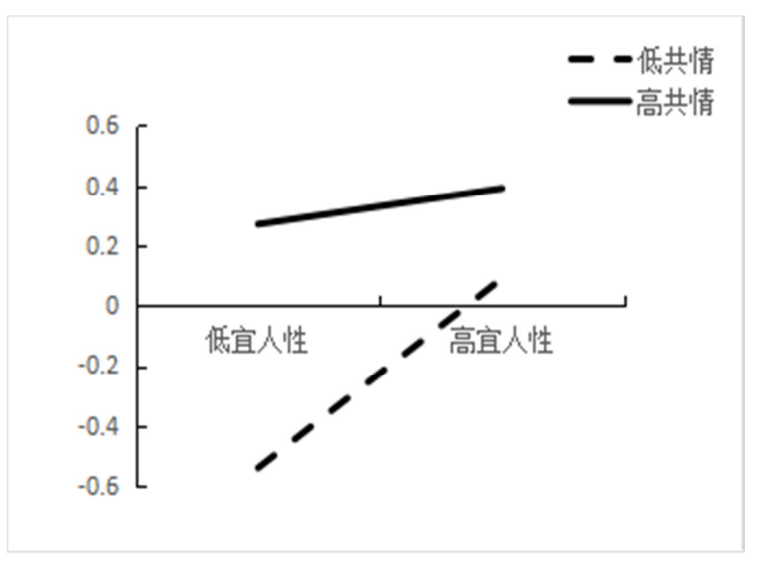

图2 共情在宜人性特质与领导力之间的调节效应。

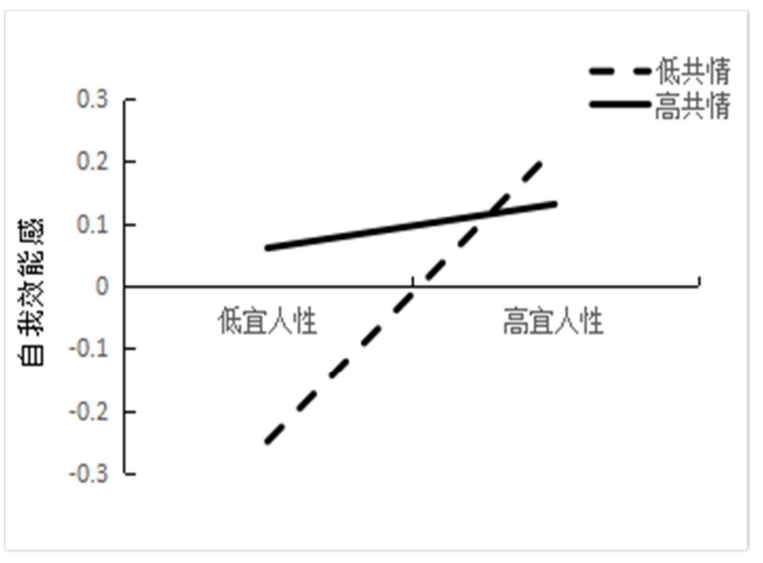

图3 共情在宜人性特质对自我效能感影响中的调节效应。

\section{4. 讨论}

\section{1. 自我效能感在宜人性特质与师范院校大学生领导力 之间的中介作用}

相关分析表明，宜人性特质对领导力有显著的正向预 测作用, 说明宜人性可以直接影响领导力, 印证了领导特 质理论。宜人性这种与生俱来的特质, 在我国更加注重团 结与和谐的文化背景下, 对领导力有着积极的影响。宜人 性特质更高的大学生, 更易表现出对他人的关心、更容易 与他人和谐相处、和表现“师爱”, 因此更容易被接纳, 从 而表现出更高的领导力, 这与前人的研究结果相似 $[21$, 22]。相反, 宜人性特质更低的大学生, 更加容易表现出 淡漠、疏离, 这与注重团结与和谐的文化背景相悖, 不能 担负起领导者协调各方的责任, 因而可能被知觉为不具有 领导能力。因此, 宜人性作为一种人格特质, 对教师领导 力有着直接的正向作用, 在我国教师选择和培养时值得引 起高度重视。

逐步回归结果显示, 自我效能感在宜人性与领导力之 间起着部分中介作用, 说明宜人性不仅可以直接影响领导 力, 而且会通过自我效能感间接影响领导力, 即宜人性可 以通过自我效能感的增强而间接地提升领导力。该结果可 以用自我效能理论来解释。在个体先天特质和生理状态一 定的情况下, 个体的效能预期会对努力程度产生影响, 使 行为主体更加主动和坚韧, 而促使绩效目标达成, 且现有
的研究也表明自我效能感的高低与领导力存在显著的相 关 [23，24]。可见自我效能感在宜人性特质与领导力之间 起着桥梁作用, 能够对领导力的激发产生积极作用。引入 自我效能感这一中介变量, 为揭示师范院校大学生宜人性 特质如何影响其领导力提供了理论和实证依据; 同时也为 师范院校大学生领导力的培养和提升方法提供了参考。

\section{2. 共情对自我效能感中介效应的调节作用}

本研究构建了一个有调节的中介效应模型, 考察了自 我效能感在宜人性特质与领导力之间的中介效应以及共 情对其关系的调节作用。结果发现, 共情对直接效应以及 中介效应的前半段路径的调节效应显著。这一有调节的中 介模型可以通过调节效应的分析, 揭示在共情的不同水平 时, 宜人性对自我效能感和领导力影响的大小。

在宜人性特质对领导力影响的直接路径中, 发现当共 情水平较低时宜人性特质对领导力的影响较大; 当共情水 平较高时, 宜人性对领导力的影响不显著。在这里好像共 情减弱了宜人性对领导力的影响, 但通过图 2 , 还是能非 常清楚地看到当共情处于高水平时, 领导力的分值还是远 远大于当共情处于低水平时的分值。也就是说, 共情能力 正向影响了宜人性与领导力之间的关系, 这可能是因为无 论宜人性高低, 高共情能力的个体的领导力都处于高水平, 所以在高共情水平时, 虽然领导力水平较高, 但宜人性对 领导力的预测作用降低。说明高共情的个体更易表现出更 高的领导力, 这与证实共情与领导力之间存在积极相关的 结果相符合[25], 也表明为提升师范院校大学生领导力, 不仅要注重人格特质的作用, 也要关注其共情能力, 在人 格特质较为稳定的情况下可以通过培养和提升共情能力 进而促进领导力培养。在中介路径中发现, 当共情水平较 低时宜人性对自我效能感的影响较大; 高共情水平时, 宜 人性对自我效能感的影响不显著。看起来也好像是共情减 弱了宜人性对领导力的影响, 但通过图3可以看到在高共 情水平时, 随着宜人性的增加, 自我效能感的分值也逐渐 上升, 只不过上升的幅度小于低共情水平, 即宜人性对自 我效能感的作用逐渐趋于稳定, 而共情对自我效能感的作 用增大。也就是说, 在宜人性特质较为稳定的情况下, 更 高的共情能力对自我效能感发挥的作用更大。而在低共情 水平时, 宜人性对自我效能感的影响增大, 此时共情这种 后天培养的能力较低, 只能通过宜人性这种特质对领导力 发挥较大作用。这说明高共情的个体更易表现出更高的自 我效能感, 从而表现出更强的领导力, 与其他表明共情影 响自我效能感的研究相一致 [26, 27]。因此, 共情能力对 自我效能感和领导力有着不可忽视的影响, 能够代替宜人 性不足而发挥正向作用, 其培养应引起足够的重视。

\section{3. 研究意义与展望}

本研究探讨了师范院校大学生宜人性特质、自我效能 感和共情三者共同对其领导力所产生的作用, 这不仅丰富 了以往有关大学生领导力的研究, 为培养和提升师范院校 学生领导力提供了理论指导, 同时也支持了领导特质理论 和自我效能感理论, 解决了以往研究中宜人性与领导力之 间关系的不确定性, 进一步表明领导者个人特质在不同文 
化背景下对领导力产生的影响以及自我效能感和共情对 领导力发挥的作用。从实践角度看, 为提升师范院校学生 未来在其职业上的领导力, 应从人格培养、效能感提升以 及共情培养的角度给予有意识地重视和指导。具体来讲, 在宜人性人格特质较为稳定的情形下, 应从多方面培养师 范院校学生的“师爱”, 如“师爱师德”课程, 使其成为更加 具有感染力、影响力的教师; 同时, 宜人性特质作为一种 较为稳定的人格特质, 在难以通过短期措施进行提升的情 况下, 可以通过自我效能感和共情的培养而实现领导力的 提升。自我效能感和共情作为一种可通过后天培养而提升 的能力对领导力产生的影响不容忽视, 学校教育可以提供 更多的支持, 如优势特长培养、团体辅导、职业发展规划、 生涯教育等, 从而增加其领导力提升的机会, 为以后在教 师职业适应和发展方面做好准备, 以发挥新时代教师的引 领作用从而实现教师与学生之间的良性互动。

本研究也存在一些不足: 第一, 采用被试主观报告法, 数据可靠性有待于进一步考察。在以后的研究中可以考虑 在实验室环境下对参与者进行测试, 控制无关变量后得到 的数据信效度可能更高; 第二, 横断研究方法无法对变量 间关系做出因果判断。因此, 未来的研究可以考虑纵向追 踪研究设计, 以便更有效的考察宜人性特质对领导力产生 影响的实质。

\section{5. 结论}

本研究主要获得以下结论:

(1) 师范院校大学生宜人性特质与领导力之间显著 正相关，与自我效能感和共情显著正相关;

(2) 自我效能感在师范院校大学生宜人性特质与领 导力之间起部分中介作用;

（3）共情在“宜人性特质 $\rightarrow$ 领导力”和“宜人性特质 $\rightarrow$ 自我效能感”两条路径上均起调节作用，即共情 可以有效增加自我效能感, 并且可以提升领导力。

\section{参考文献}

[1] Hashem, M. (1997). The role of faculty in teaching leadership studies. Journal of Leadership Studies, 4(2), 89-100.

[2] Tsai, F., \& Chu, L. (2014). Research on the cultivation of the leadership ability: From the point of strategic leadership. Jiaoyu Yanjiu Yuekan = Journal of Education Research, (246), 40-56.

[3] Wang, X. P. (2010). Study of leadership consciousness of college students cultivation (Order No. 10534310).

[4] 张超.(2012)大学生领导力: 结构及相关影响因素的路径分 析.(Doctoral dissertation, 华东师范大学)。

[5] 房欲飞.(2008). 大学生领导力教育研究述评.现代教育科学 (2), 153-155+158.

[6] 林文曼.(2019). 师范院校大学生的领导力培养研究. 西部素 质教育,5(10):82-83。
[7] 彭云.(2017).教师领导力的核心要素与提升路径.教育理论 与实践(23),29-31。

[8] 姚荻琳,郑俊巍, 宋炳良.(2019). 教师领导力对大学生学习 投入影响的实证研究.大学教育(10),186-189。

[9] 王丹玮,王艺.(2019).教师领导力对师生关系发展的影响及 对策研究.智库时代, 180(12),274-275。

[10] 连灵, 郭胜忠. (2017). 大学生宜人性和心理幸福感的关系: 领悟社会支持和感恩的链式中介作用.中国临床心理学杂 志,25(01):163-166。

[11] Terman, L. M. (1904). A preliminary study in the psychology and pedagogy of leadership. Journal of Genetic Psychology, $11,413-451$.

[12] Judge, T. A., Bono, J. E., Ilies, R., \& Gerhardt, M. W. (2002). Personality and leadership: A qualitative and quantitative review. Journal of Applied Psychology, 87(4), 765-780.

[13] 王超,袁蒙蒙,姜媛,方平.(2019). 宜人性对企业家成就的影响: 来自元分析的证据.心理与行为研究, 17(01), 128-135。

[14] 乔刘成,刘树涁,路亚男,胡维芳.(2017). 高职教师人格特征与 教学效能感的关系研究.职教通讯(29),46-50+54。

[15] 丁瑾哂.(2018). 大学生人格特质与创业意向的关系:自我效 能感的中介效应. 呼伦贝尔学院学报, 26(03), 128-133。

[16] Bandura, A. (in press). The self and mechanisms of agency. In J. Suls (Ed.), Social psychological perspectives on the self. Hillsdale, N.J.: Erlbaum, (in press).

[17] 周炎根,张鹏程, 张岩.(2018).大学生的内控归因和共情在 宜人性与宽恕关系中的多重中介效应.中国心理卫生杂 志,32(7), 90-94。

[18] 郑显亮,赵薇.(2015).共情、自我效能感与网络利他行为的关 系.中国临床心理学杂志, 23(2), 358-361。

[19] Yazid, A., Bakar, A., Mohd, N., Hakimie, M., \& Abidin, Z. (2014). The relationship between domains of empathy and leadership skills among gifted and talented students. Procedia - Social and Behavioral Sciences, 116, 765-768.

[20] 温忠麟, 张雷,侯杰泰.(2006). 有中介的调节变量和有调节的 中介变量. 心理学报,38(3), 448-452。

[21] 范竞宇.(2019). 性格宜人性对领导力的影响研究. 现代商贸 工业,40(06),77-79。

[22] 墽知仁.(2018). 宜人性对领导力的影响以及性别差异. 科技 经济导刊,26(31), 179-181。

[23] Sun, A., \& Xia, J. (2018). Teacher-perceived distributed leadership, teacher self-efficacy and job satisfaction: A multilevel SEM approach using the 2013 TALIS data. International Journal of Educational Research, 92(April), 8697.

[24] Qiu, S., Dooley, L. M., \& Xie, L. (2019). How servant leadership and self-efficacy interact to affect service quality in the hospitality industry: A polynomial regression with response surface analysis. Tourism Management, 78, 104051. 
[25] Sadri, G., Weber, T. J., \& Gentry, W. A. (2011). Empathic emotion and leadership performance: An empirical analysis across 38 countries. The Leadership Quarterly, 22(5), 818830.

[26] Goroshit, M., \& Hen, M. (2016). Teachers' empathy: Can it be predicted by self-efficacy? Teachers and Teaching: Theory and Practice, 22(7), 805-818.
[27] Flickinger, T. E., Saha, S., Roter, D., Korthuis, P. T., Sharp, V., Cohn, J. \& Catherine, M. (2016). Patient Education and Counseling Clinician empathy is associated with differences in patient - clinician communication behaviors and higher medication self-ef fi cacy in HIV care. Patient Education and Counseling, 99(2), 220-226. 